\title{
DSC as a valuable tool for the evaluation of adulteration of oils and fats
}

\begin{abstract}
Authentication is part of quality assessment to maintain purity standards in oils and fats. It is highly useful for oils and fats as it helps to combat adulteration practices taking place in trade and industries. Among the different analytical techniques, DSC is a valuable tool for establishing identity of pure oils and fats for authentication purposes. It is nondestructive technique and works with minimal sample preparation. DSC analyses of palmitic, lauric, and oleic oils can provide well-defined thermal curves, which can be compiled as a data base to become a reference for investigations leading to detection of adulterations. As DSC curves of plant oils and fats differ considerably from those of animal fats, adulterations in plant oils and fats caused by animal fats can be detected easily. There is basis for detection of adulteration qualitatively, if significant deviations are noticed in the DSC curve of a particular oil or fat with respect to that of the reference authentic sample. However, based on the characteristic composition of individual oil and fat, the detection approaches and limits of detection for either LD or BF differ considerably. DSC could also be used effectively for quantification of adulterations as DSC variables associated with adulteration peaks were found to show high correlation with the increasing levels of adulteration. For most oils, predictive models for estimation of lard adulteration can be obtained using stepwise multiple linear regression (SMLR) analysis with \% LD (adulteration level) as the dependent variable and DSC parameters (peak area, A; peak height, HT; and peak onset, ON) of adulteration peak as independent variables.
\end{abstract}

